\title{
Primary squamous cell carcinoma of thyroid: a case report and review of literature
}

Mutahir A Tunio ${ }^{1 *}$, Mushabbab Al Asiri , Mosa Fagih² and Rashad Akasha ${ }^{3}$

\begin{abstract}
Background: Thyroid gland lacks squamous epithelium (except in some rare situations like embroyonic remnants or in inflammatory processes); for that reason the primary squamous cell carcinoma (SCC) of thyroid is extremely rare entity, seen only in less than $1 \%$ of all thyroid malignancies and is considered almost fatal. So, far, only few case reports have been published in literature.

Case presentation: Herein we present a 54 years old Saudi female with 3 months history of progressive neck swelling and hoarse voice, who was referred to us by her primary care physician as suspected case of anaplastic carcinoma of thyroid for radical external beam radiation therapy (EBRT). Fine Needle aspiration cytology (FNAC) revealed squamous cell carcinoma. Computed tomography $(C T)$ neck showed $10 \times 10 \mathrm{~cm}$ mass in left lobe of thyroid invading trachea and skin. Extensive staging work up ruled out the possibility of any primary site of SCC other than thyroid gland. Tumor was found unresectable and was referred to radiation oncology. She received palliative EBRT $30 \mathrm{~Gy}$ in 10 fractions. After completion of EBRT, there was progression of disease and patient died 3 months after completion of EBRT by airway compromise.

Conclusion: Primary SCC of thyroid is rare and aggressive entity. FNAC is reliable and effective tool for immediate diagnosis. Surgery is a curative option, but it is not always possible as most of cases present as locally advanced with adjacent organs involvement. EBRT alone was found ineffective. Aggressive combined modality (debulking surgery, radiation and chemotherapy) shall be considered for such cases.
\end{abstract}

Keywords: Squamous cell carcinoma, Thyroid, Rare, Primary, Fatal

\section{Background}

Primary squamous cell carcinoma (SCC) of thyroid is an uncommon malignancy and has poor prognosis [1]. SCC of thyroid constitutes less than $1 \%$ of thyroid malignancies and has been found fatal within one year of initial diagnosis [2]. The median age is fifth and sixth decade, but can be seen at any age. Main cause of death in these patients is secondary to respiratory interference by direct invasion or compression of the trachea [3]. When SCC of thyroid is diagnosed, the possibility of the tumor arising from adjacent organs (esophagus, larynx) or representing metastatic disease from primary growth somewhere else (lungs) must be considered before concluding the malignancy as SCC of thyroid.

\footnotetext{
* Correspondence: drmutahirtonio@hotmail.com

${ }^{1}$ Department of Radiation Oncology, Comprehensive Cancer Center, King

Fahad Medical City, Riyadh, PO 59046, Saudi Arabia

Full list of author information is available at the end of the article
}

The etiology of SCC thyroid is uncertain as thyroid gland lacks the squamous epithelium. However three theories have been postulated; first the embryonic nest theory suggests that squamous cells are derived from the embryonic remnants such thyroglossal duct, thymic epithelium and ultimobronchial body [4]. Second the metaplasia theory suggests that the environmental stimuli (inflammation and Hashimoto's thyroiditis) result in squamous metaplasia [5]. Third the de-differentiation theory suggests that existing papillary, follicular, medullary and anaplastic thyroid carcinoma de-differentiate into SCC [6,7].

Herein we present a case of 54 years old Saudi lady with locally advanced primary squamous cell carcinoma of thyroid, diagnosed by fine needle aspiration cytology (FNAC) was treated with radiation therapy.

\section{Case presentation}

A 54 year old Saudi female presented in our clinic with neck swelling and hoarse voice. She had noticed this

\section{() Biomed Central}


swelling for 3 months and it had been rapidly increasing in size over a week causing dyspnoea and dysphagia to solids. Her previous medical history revealed type II diabetes mellitus since last 10 years and hypothyroidism since last 3 years, for that she was taking thyroxin 50 micrograms daily and metformin. She had no history of smoking and her weight was stable.

On physical examination, her vitals were stable. A fixed hard neck mass of size $8 \times 8 \mathrm{~cm}$ was palpable in the left thyroid lobe with inflammatory surface Figure 1. There was no palpable cervical lymphadenopathy and examination of chest, heart, nervous system and abdomen was normal. Clinical differential diagnosis was anaplastic carcinoma of thyroid.

Ultrasonography showed huge left thyroid lobe partially cystic and solid mass of size $8.5 \times 9 \mathrm{~cm}$. Computed tomography $(\mathrm{CT})$ neck showed $10 \times 10 \mathrm{~cm}$ mass in left lobe of thyroid, partially necrotic invading to adjacent skin and trachea and no lymphadenopathy was found Figure 2. Serum T4, thyroid stimulating hormone $(\mathrm{TSH})$, thyroglobulin and serum calcium were within normal limits. Fine needle aspiration cytology (FNAC) of mass was performed, which revealed squamous cell carcinoma Figure 3. Differential diagnosis was metastatic

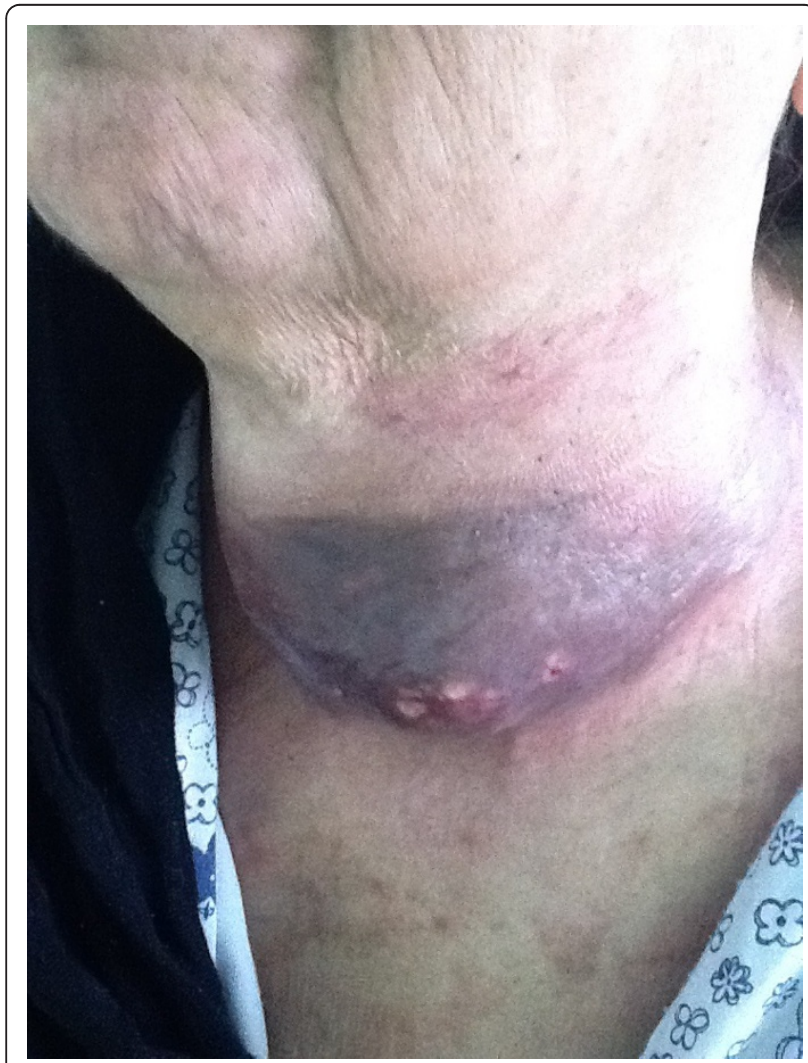

Figure $1 \mathrm{~A}$ fixed hard neck mass of size $8 \times 8 \mathrm{~cm}$ was palpable in the left thyroid lobe with inflammatory surface.

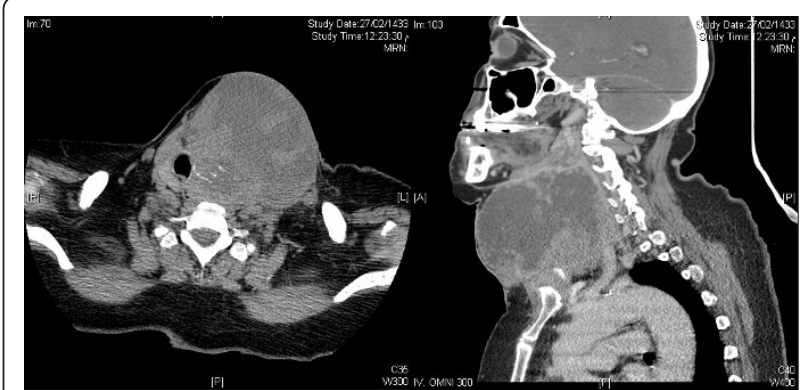

Figure 2 Computed tomography (CT) neck showing $10 \times 10$ $\mathrm{cm}$ mass in left lobe of thyroid, partially necrotic invading to adjacent skin and trachea and no cervical lymphadenopathy.

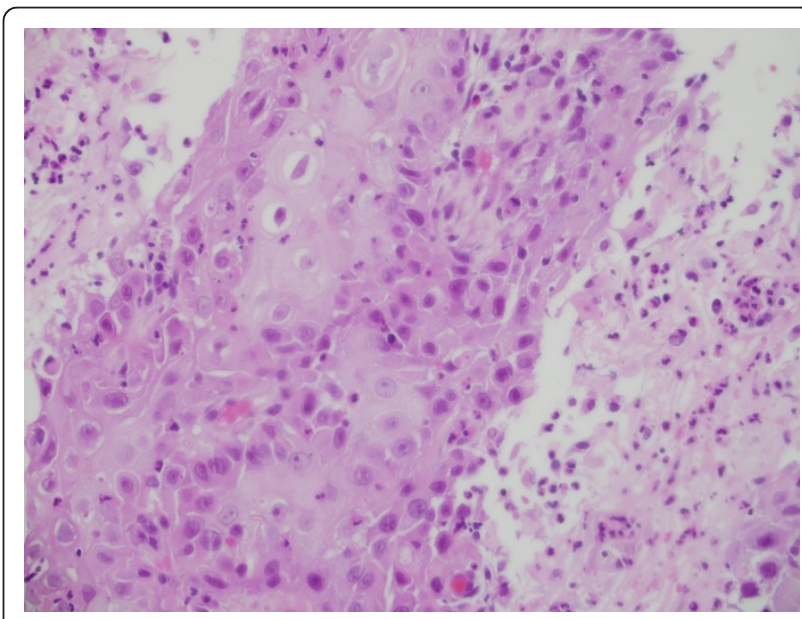

Figure 3 Fine needle aspiration cytology (FNAC) showing nests of pleomorphic cells with abundant eosinophilic cytoplasm and keratin formation along with intercellular bridging.

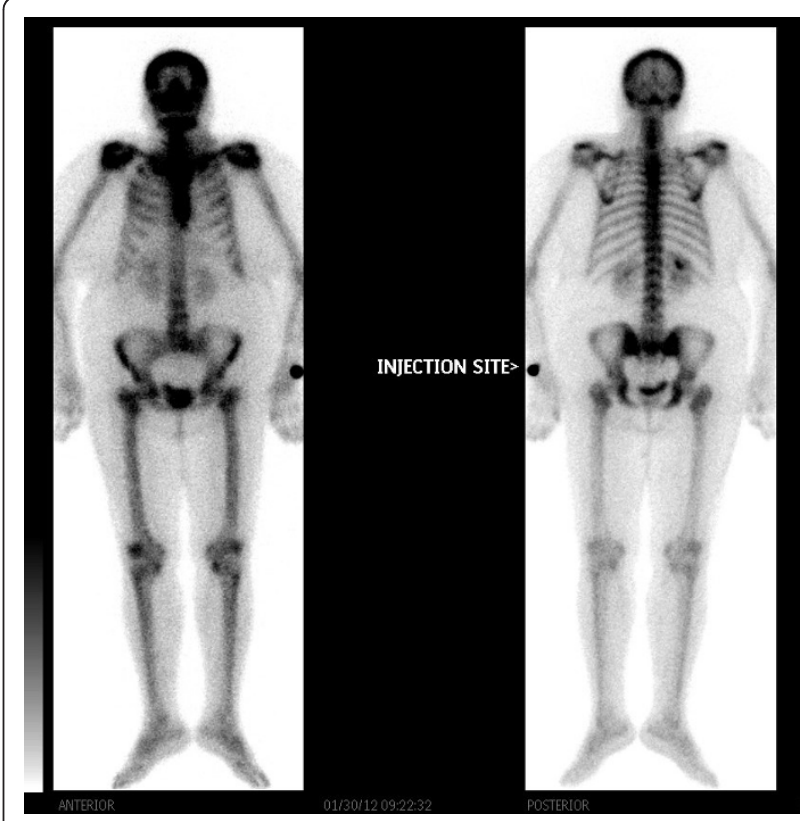

Figure 4 Bone scintigraphy showing no evidence of distant bone metastasis. 
Table 1 Previously published case reports (2000-2012) of primary squamous cell carcinoma of thyroid

\begin{tabular}{|c|c|c|c|c|c|c|c|}
\hline Author [Ref] & Gender/Age & Presentation & Stage & $\begin{array}{l}\text { Associated } \\
\text { Problem }\end{array}$ & Treatment given & Survival & Comments \\
\hline $\begin{array}{c}\text { Zimmer PW } \\
{[1]}\end{array}$ & $\begin{array}{c}\text { Female/64 } \\
\text { years }\end{array}$ & $\begin{array}{l}\text { Asymptomatic neck } \\
\text { mass }\end{array}$ & T2NOMO & - & Total thyroidectomy & $\begin{array}{c}7 \\
\text { months }\end{array}$ & - \\
\hline Kebapci N [7] & $\begin{array}{c}\text { Female/25 } \\
\text { years }\end{array}$ & Right neck mass & T4N1M0 & $\begin{array}{l}\text { Hashimotos' } \\
\text { thyroiditis }\end{array}$ & & & \\
\hline $\begin{array}{c}\text { Papillary } \\
\text { carcinoma }\end{array}$ & $\begin{array}{l}\text { Total } \\
\text { thyroidectomy } \\
\text { and RAl } \\
\text { therapy }\end{array}$ & 44 months & - & & & & \\
\hline Ko YS [8] & Male/87 years & $\begin{array}{l}\text { Asymptomatic neck } \\
\text { mass }\end{array}$ & T4NOMO & - & Right lobectomy & NA & $\begin{array}{c}\text { CK5/6 + } \\
\text { CK19 + } \\
\text { EMA,p53 focal + } \\
\text { BRAF mutation + }\end{array}$ \\
\hline $\begin{array}{l}\text { Mercante G } \\
\text { [9] }\end{array}$ & Male/67 years & - & T2NOMO & $\begin{array}{l}\text { Follicular } \\
\text { carcinoma }\end{array}$ & $\begin{array}{l}\text { Lobe-isthmusectomy + } \\
\text { Adjuvant chemoradiation }\end{array}$ & 2 years & - \\
\hline $\begin{array}{c}\text { De Vos FY } \\
\text { [10] }\end{array}$ & - & Neck mass & T4NOMO & - & $\begin{array}{l}\text { Induction chemotherapy } \\
\text { (Cisplatin + paclitaxel) }\end{array}$ & & \\
\hline $\begin{array}{c}\text { Total } \\
\text { thyroidectomy }\end{array}$ & 20 months & $\begin{array}{l}\text { Induction } \\
\text { chemotherapy } \\
\text { resulted in R0 } \\
\text { resection }\end{array}$ & & & & & \\
\hline Yucel H [11] & Male/88 years & Neck mass & T4NOMO & Hyperthyroidism & $\begin{array}{l}\text { Total thyroidectomy } \\
\text { Adjuvant radiation therapy }\end{array}$ & $\begin{array}{c}6 \\
\text { months }\end{array}$ & $\begin{array}{c}\text { Patient RAl therapy } 20 \\
\text { years back }\end{array}$ \\
\hline Eorn TI [12] & $\begin{array}{c}\text { Female/43 } \\
\text { years }\end{array}$ & Neck mass & T3NOMO & $\begin{array}{l}\text { Papillary } \\
\text { carcinoma }\end{array}$ & $\begin{array}{l}\text { Total thyroidectomy } \\
\text { Adjuvant radiation therapy } \\
59.4 \mathrm{~Gy} \\
\text { And RAl }\end{array}$ & $\begin{array}{c}8 \\
\text { months }\end{array}$ & $\begin{array}{l}\text { CK7 + } \\
\text { p } 63+\end{array}$ \\
\hline Makay O [13] & $\begin{array}{l}\text { Male/53 years } \\
\text { Male/71 years }\end{array}$ & $\begin{array}{l}\text { Neck mass, hoarse } \\
\text { voice and weight } \\
\text { loss }\end{array}$ & T3NOMO & - & $\begin{array}{c}\text { Near total thyroidectomy } \\
\text { Chemoradiation } 50 \mathrm{~Gy}+ \\
\text { Doxorubicin and } \\
\text { cyclophosphamide }\end{array}$ & $\begin{array}{c}2 \\
\text { months } \\
4 \\
\text { months } \\
5 \\
\text { months }\end{array}$ & - \\
\hline Fassan M [17] & $\begin{array}{l}\text { Female/64 } \\
\text { years }\end{array}$ & Neck mass & T3NOMO & Goiter & Total thyroidectomy & NA & $\begin{array}{l}\text { CK } 5 / 6+ \\
\text { CK } 7+ \\
\text { CK } 19+\end{array}$ \\
\hline $\begin{array}{c}\text { Maamouri F } \\
{[18]}\end{array}$ & $\begin{array}{c}\text { Female/87 } \\
\text { years }\end{array}$ & Right neck mass & T3NOMO & $\begin{array}{l}\text { Papillary } \\
\text { carcinoma }\end{array}$ & $\begin{array}{l}\text { Total thyroidectomy } \\
\text { And RAl therapy }\end{array}$ & $\begin{array}{c}6 \\
\text { months }\end{array}$ & - \\
\hline $\begin{array}{c}\text { Chintamani } \\
{[14]}\end{array}$ & $\begin{array}{c}\text { Female/50 } \\
\text { years } \\
\text { Male/60 years } \\
\text { Male/58 years }\end{array}$ & $\begin{array}{l}\text { Dysphagia, hoarse } \\
\text { voice and stridor }\end{array}$ & T4NOMO & Hyperthyroidism & $\begin{array}{c}\text { Total thyroidectomy } \\
\text { Adjuvant radiotherapy } 50 \\
\text { Gy }\end{array}$ & $\begin{array}{c}12 \\
\text { months }\end{array}$ & - \\
\hline Jung TS [15] & Male/56 years & $\begin{array}{l}\text { Neck mass, hoarse } \\
\text { voice }\end{array}$ & T3NOMO & $\begin{array}{l}\text { Follicular } \\
\text { carcinoma }\end{array}$ & $\begin{array}{c}\text { Total thyroidectomy } \\
\text { Adjuvant radiotherapy } 50 \\
\text { Gy } \\
\end{array}$ & 8 years & - \\
\hline Sutak J [19] & $\begin{array}{c}\text { Female/ } 80 \\
\text { years }\end{array}$ & $\begin{array}{l}\text { Asymptomatic neck } \\
\text { mass }\end{array}$ & T4N1M0 & $\begin{array}{c}\text { Tall cell variant } \\
\text { papillary } \\
\text { carcinoma }\end{array}$ & Total thyroidectomy & - & $\begin{array}{c}\text { CK } 7+ \\
\text { CK } 19+ \\
\text { CK AE1/3+ } \\
\text { P53 focal + }\end{array}$ \\
\hline Zhou XH [16] & 4 patients & NA & T4NOMO & - & $\begin{array}{c}\text { Total thyroidectomy } \\
\text { Adjuvant radiotherapy } 50 \\
\text { Gy + chemotherapy }\end{array}$ & $\begin{array}{c}4 \\
\text { months } \\
6 \\
\text { months } \\
13 \\
\text { months } \\
26 \\
\text { months }\end{array}$ & $\begin{array}{l}\text { Longer survival was } \\
\text { seen in combined } \\
\text { trimodality treatment }\end{array}$ \\
\hline Lam KY [20] & $\begin{array}{c}4 \text { females/71 } \\
\text { years }\end{array}$ & $\begin{array}{l}\text { Neck mass, } \\
\text { stridor }\end{array}$ & T4NOMO & - & Total thyroidectomy & $\begin{array}{c}4 \\
\text { months }\end{array}$ & $\begin{array}{c}\text { CK } 7+ \\
\text { CK } 19+ \\
\text { CK AE1/3+ } \\
\text { P53 focal + }\end{array}$ \\
\hline
\end{tabular}


Table 1 Previously published case reports (2000-2012) of primary squamous cell carcinoma of thyroid (Continued)

\begin{tabular}{|c|c|c|c|c|c|c|c|}
\hline Kleer CG [21] & $\begin{array}{c}7 \text { females } / 1 \\
\text { male } \\
31-90 \text { years }\end{array}$ & Neck mass & T4NOMO & $\begin{array}{c}\text { Tall cell variant } \\
\text { papillary } \\
\text { carcinoma }\end{array}$ & Total thyroidectomy & $\begin{array}{c}6 \\
\text { Months- } \\
48 \\
\text { months }\end{array}$ & - \\
\hline Jones JM [22] & Male/48 years & $\begin{array}{l}\text { Hoarse voice, } \\
\text { left neck mass }\end{array}$ & T4N1M0 & - & $\begin{array}{c}\text { Total thyroidectomy and } \\
\text { LND }\end{array}$ & $\begin{array}{c}8 \\
\text { months }\end{array}$ & - \\
\hline
\end{tabular}

squamous cell carcinoma from another primary location. CT chest, abdomen, pelvis, magnetic resonance imaging (MRI) of head and neck region, pan-endoscopy, laryngoscopy, esophagoscopy and bone scintigraphy did not reveal any primary lesion or other metastatic disease Figure 4. Radiological stage was made as T4N0M0.

In a multidisciplinary tumor (MDT) meeting it was labeled unresectable and patient was referred for external beam radiation therapy (EBRT) after prophylactic percutaneous endoscopic gastrostomy (PEG) insertion. Due to retrosternal extention of disease, tracheostomy was deferred. Patient received 30 Grays (Gy) in 10 fractions to thyroid. Post radiation therapy, there was progression size of neck mass with progressive dyspnoea. Patient died of airway compromise 3 months of palliative EBRT.

\section{Discussion}

Primary SCC of the thyroid gland is an extremely rare and aggressive entity usually presents with classic triad features; (I) rapidly enlarging mass in the older patients behaving like anaplastic carcinoma, (II) it may be associated with other thyroid malignancies and (III) histological features of intercellular bridges and keratin [8-10]. FNAC is reliable and confirmatory tool, but it is mandatory to exclude the metastatic SCC [11].

Treatment with surgery, radiation therapy and chemotherapy alone has been found ineffective in previously published similar case reports, as majority of these patients present as locally advanced cases not amenable for curative resection Table 1 . The better survival rates have been achieved with aggressive combination therapy (surgery followed by adjuvant radiation therapy (50-60 Gy) with or without chemotherapy or induction chemotherapy followed by surgery) [11-16].

\section{Conclusion}

Primary squamous cell carcinoma of thyroid is a rare and aggressive entity with poor prognosis. FNAC is effective confirmatory tool, but efforts shall be made to rule out metastatic SCC originating from other sites. Surgery, radiotherapy and chemotherapy alone are ineffective. Aggressive treatment with surgery followed by adjuvant radiotherapy with or without chemotherapy is recommended to achieve better outcome.

\section{Consent}

Written permission was taken from the patient for publication of the case report.

\section{Abbreviations}

SCC: Squamous cell carcinoma; EBRT: External beam radiation therapy; FNAC: Fine needle aspiration cytology; CT: Computed tomography; RAl: Radioactive iodine; TSH: Thyroid stimulating hormone; MDT: Multidisciplinary tumor meeting; PEG: Percutaneous endoscopic gastrostomy.

\section{Author details}

${ }^{1}$ Department of Radiation Oncology, Comprehensive Cancer Center, King Fahad Medical City, Riyadh, PO 59046, Saudi Arabia. ${ }^{2}$ Department of Cytogenetics, King Fahad Medical City, Riyadh, PO 59046, Saudi Arabia. ${ }^{3}$ Resident Radiation Oncology, Department of Radiation Oncology, Comprehensive Cancer Center, King Fahad Medical City, Riyadh, PO 59046, Saudi Arabia.

\section{Authors' contributions}

MAT, MAA Manuscript preparation. RA Data Collection. MF Pathological data. All authors read and approved the final manuscript.

\section{Competing interests}

Authors have neither potential conflict of interest nor received any grants for this case report.

Received: 28 February 2012 Accepted: 27 March 2012

Published: 27 March 2012

\section{References}

1. Zimmer PW, Wilson D, Bell N: Primary squamous cell carcinoma of the thyroid gland. Mil Med 2003, 168:124-5.

2. Korovin GS, Cho HT, Kuriloff DB, Sobol SM: Squamous cell carcinoma of the thyroid: a diagnostic dilemma. Ann Otol Rhinol Laryngol 1989, 98:59-65.

3. Simpson WJ, Carruthers TH: Squamous cell carcinoma of thyroid gland. Am J Surg 1988, 156:44-6.

4. Goldberg HM, Harrey P: Squamous cell cysts of the thyroid with special reference to the etiology of squamous epithelium in the human thyroid. $\mathrm{Br} J$ Surg 1956, 43:565-9.

5. Chaudhary RK, Barnes EL, Myers EN: Squamous cell carcinoma arising in Hashimoto's thyroiditis. Head Neck 1994, 16:582-5.

6. Bronner MP, LiVolsi VA: Spindle cell squamous carcinoma of the thyroid: an unusual anaplastic tumor associated with tall cell papillary carcinoma. Mod Pathol 1991, 4:630-43.

7. Kebapci N, Efe B, Kabukcuoglu S, Akalin A, Kebapci M: Diffuse sclerosing variant of papillary thyroid carcinoma with papillary squamous cell carcinoma. J Endocr Invest 2002, 25:730-4.

8. Ko YS, Hwang TS, Han HS, Lim SD, Kim WS, Oh SY: Primary pure squamous cell carcinoma of the thyroid: report and histogenic consideration of a case involving a BRAF mutation. Pathol Int 2012, 62:43-8

9. Mercante G, Marchesi A, Covello R, Dainese L, Spiano G: Mixed squamous cell carcinoma and follicular carcinoma of the thyroid gland. Auris Nasus Larynx 2011, 8:1-5, PMID 21855238.

10. De Vos FY, Sewnaik A, de Witt JH, Smid EJ, den Bakker MA, van Meerten E: Combined therapy for thyroid squamous cell carcinoma. Head Neck 2012, 34:131-4. 
11. Yucel H, Schaper NC, van Beek M, Bravenboer B: Primary squamous cell carcinoma of the thyroid years after radioactive iodine treatment. Neth J Med 2010, 68:224-6.

12. Eorn TI, Koo BY, Kim BS, Kang KH, Jung SK, Jun SY, Bae HS, Kim LS: Coexistence of primary squamous cell carcinoma of thyroid with classic papillary thyroid carcinoma. Pathol Int 2008, 58:797-800

13. Makay O, Kaya T, Ertan T, Icoz G, Akyildiz M, Yilmaz M, Tuncyurek M, Yetkin E: Primary squamous cell carcinoma of the thyroid: report of three cases. Endocr J 2008, 55:359-64.

14. Chintamani PK, Singh J, Sugandhi N, Bansal A, Bhatnagar D, Saxena S: Is an aggressive approach justified in the management of an aggressive cancer- the squamous cell carcinoma of thyroid? Int Semin Surg Oncol 2007, 4:8.

15. Jung TS, Oh YL, Min YK, Lee MS, Lee MK, Kim KW, Chung JH: Korean J Intern Med 2006, 21:73-8.

16. Zhou XH: Primary squamous cell carcinoma of the thyroid. Eur I Surg Oncol 2002, 28:42-5.

17. Fassan M, Pennelli G, Pelizzo MR, Rugge M: Primary squamous cell carcinoma of the thyroid. Immunohistochemical profile and literature review. Tumori 2007, 93:518-21.

18. Maamouri F, Goucha A, Ben Mna N, Ben Hassouna J, Debbabi B, Ouslati Z, Boussen H, El May A, Gamoudi A: Tunis Med 2007, 85:251-3.

19. Sutak J, Armstrong JS, Rusby JE: Squamous cell carcinoma arising in a tall cell papillary carcinoma of the thyroid. J Clin Pathol 2005, 58:662-4.

20. Lam KY, Lo CY, Liu MC: Primary squamous cell carcinoma of thyroid gland: an entity with aggressive clinical behavior and distinctive cytokeratin expression profiles. Histopathology 2001, 39:279-86.

21. Kleer CG, Giordano TJ, Merino MJ: Squamous cell carcinoma of the thyroid: an aggressive tumor associated with tall cell variant of papillary thyroid carcinoma. Mod Pathol 2000, 13:742-6.

22. Jones JM, McCluggage WG, Russell CF: Primary squamous cell carcinoma of the thyroid. Ulster Med J 2000, 69:58-60.

doi:10.1186/1758-3284-4-8

Cite this article as: Tunio et al:: Primary squamous cell carcinoma of thyroid: a case report and review of literature. Head \& Neck Oncology 2012 4:8.

\section{Submit your next manuscript to BioMed Central and take full advantage of:}

- Convenient online submission

- Thorough peer review

- No space constraints or color figure charges

- Immediate publication on acceptance

- Inclusion in PubMed, CAS, Scopus and Google Scholar

- Research which is freely available for redistribution

Submit your manuscript at www.biomedcentral.com/submit 